\title{
Von steinzeitlichen Heilpflanzen und wurmbefallenem Kot
}

\section{Emanuela Jochum Zimmermann}

lic. phil. I, Archäologin und Ausstellungskoordinatorin

Sie wollten schon immer jahrtausendealten Kot sehen und wissen, wie Steinzeitmenschen Schädeloperationen durchgeführt haben? Die interaktive Ausstellung «Gesundheit - 7000 Jahre Heilkunst» im Kulturama Museum des Menschen in Zürich fasst Erkenntnisse aus Archäobotanik, Parasitologie, Anthropologie, Virologie, Paläogenetik und Medizingeschichte zusammen. Sie nimmt Laien und Fachleute mit auf eine Reise durch die Medizingeschichte seit der Steinzeit.

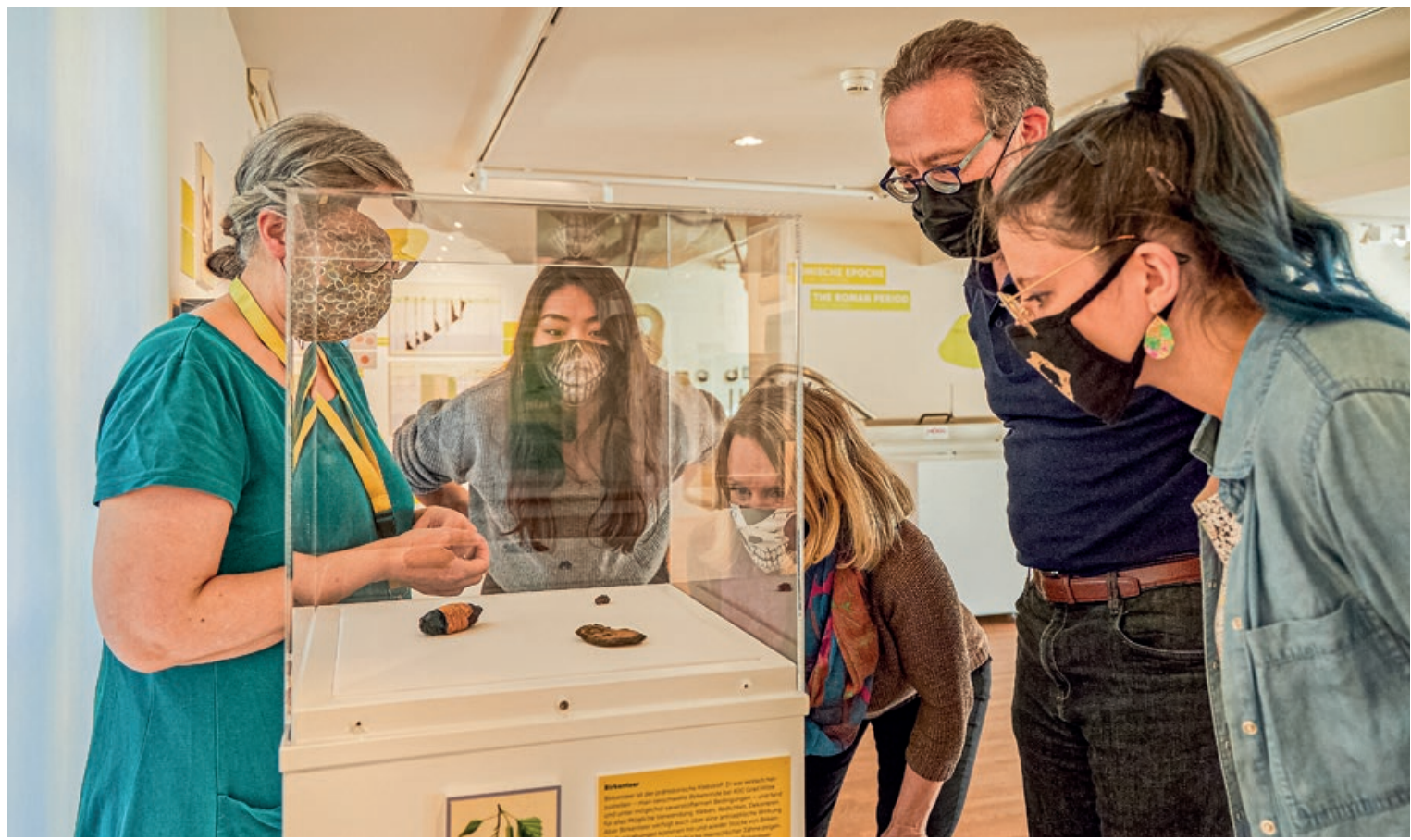

Interaktive Ausstellung über die Geschichte der Medizin - von der Steinzeit bis heute (๔ Kulturama, Joanna Lesniewska).

Die Ausstellung wurde konzipiert von der

Kantonsarchäologie Luzern und dem Museum für Urgeschichte(n) Zug mit Erweiterungen des Kulturama Museum des Menschen.
Die Ausstellung behandelt zwei grosse Themenbereiche: die Anthropologie und die Archäobotanik. Knochenfunde und anthropologische Untersuchungen zeigen beispielhaft, an welchen Krankheiten und Verletzungen Menschen früher litten. Im Boden erhalten sich menschliche Knochen unter guten Bedingungen Jahrtausende. Sie erlauben Rückschlüsse auf Alter, Geschlecht und Körperbau einer verstorbenen Person. Mangelernährung, Krankheiten, und sogar Behandlungsmethoden oder Verletzungen wie Knochenbrü- che oder Schwerthiebe hinterlassen Spuren an den Knochen [1]. Ergebnisse verschiedener archäobotanischer Forschungen dokumentieren, wie Heilpflanzen auf archäologischen Ausgrabungen nachgewiesen werden sowie deren Nutzung von der Jungsteinzeit bis in die Neuzeit [2]. Für die Erhaltung von Pflanzenresten und Parasiten sind nur wenige Bedingungen ideal. In feuchtem Milieu und unter Luftabschluss haben sich die gezeigten Funde aus den Seeufersiedlungen erhalten. 


\section{Frühe Pflanzenheilkunde}

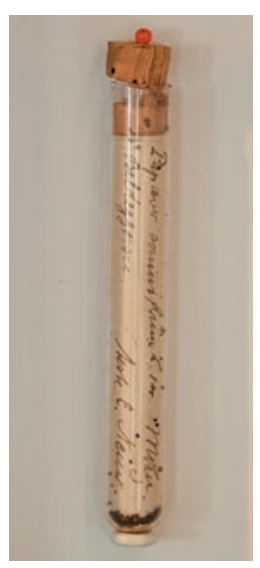

Mohnsamen, ZugSumpf ZG, Siedlung, 1050-870 v. Chr. (C) Kulturama, Joanna Lesniewska).
Schon in der Jungsteinzeit und Bronzezeit verfügten die Menschen über eine profunde Kenntnis der Natur und Wissen über die Heilwirkung von Pflanzen [3]. Samen in Pfahlbausiedlungsschichten zeigen, dass gezielt Pflanzen mit Heilwirkungen gesammelt oder vereinzelt auch auf dem Acker angepflanzt wurden. Dazu gehört zum Beispiel der opiumhaltige Schlafmohn. Sehr wahrscheinlich wurden viele der Pflanzen, denen später von der Volksmedizin eine heilende Wirkung zugeschrieben wurde, schon damals als Heilpflanzen eingesetzt [4].

\section{Keimfalle Pfahlbaudorf}

Das Leben im Pfahlbaudorf war nicht romantisch. Das dichte Zusammenleben im Dorf und die harte Arbeit hatten Folgen: Abnützungserscheinungen, Verletzungen, Karies, Entzündungen und Parasiten gehörten zum Alltag. Fast die Hälfte der Kinder wurde keine fünf Jahre alt. Wer das Erwachsenenalter erreichte, hatte

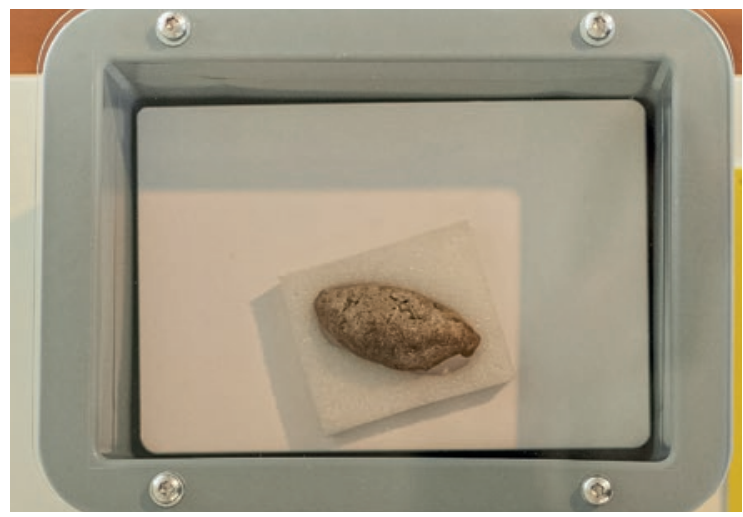

Menschlicher Kotrest, Arbon-Bleiche TG, 3380 v. Chr. (C) Kulturama, Joanna Lesniewska).

\section{Angebote für Gruppen}

Zur Sonderausstellung "Gesundheit - 7000 Jahre Heilkunst» bietet das Kulturama Museum des Menschen zielgruppengerechte Führungen an (in Deutsch, Englisch und Spanisch). Die Ausstellung läuft bis zum 30. April 2022.

Alle Angaben zu Führungen und Öffnungszeiten finden Sie unter:

Kulturama Museum des Menschen

www.kulturama.ch

mail[at]kulturama.ch

+41442606044 eine Lebenserwartung von nur etwa 40 Jahren [5]. Die organischen Fundschichten enthalten nicht nur Siedlungsabfall, sondern auch Mist und menschliche Exkremente. Unter günstigen Bedingungen bleibt selbst die Form der Kotreste erhalten. Darin lassen sich zahlreiche Darmparasiten nachweisen: Bandwurm, Peitschenwurm, Leberegel, Fischbandwurm, Nierenwurm und Haarwurm. Doch ein spannender Fund zeigt: Die Menschen wussten sich zu helfen. Ein in der Ausstellung präsentierter Kotrest aus Arbon weist Sporen des Wurmfarns auf, einer Pflanze, die bis in historische Zeit gegen Würmer eingesetzt wurde.

\section{Operation am offenen Schädel}

Eindrückliche medizinische Kenntnisse der Steinzeit dokumentieren die Trepanationen, also operativen Öffnungen der Schädeldecke. Erstaunlicherweise überlebte der Patient oder die Patientin diese Behandlung häufig. Grosse Schmerzen und Infektionen wurden offenbar bekämpft. Welche Leiden so behandelt wurden, ist unbekannt: Waren es konkrete physische Krankheiten und Verletzungen - oder musste etwas «Böses» aus dem Kopf herausgelassen werden?

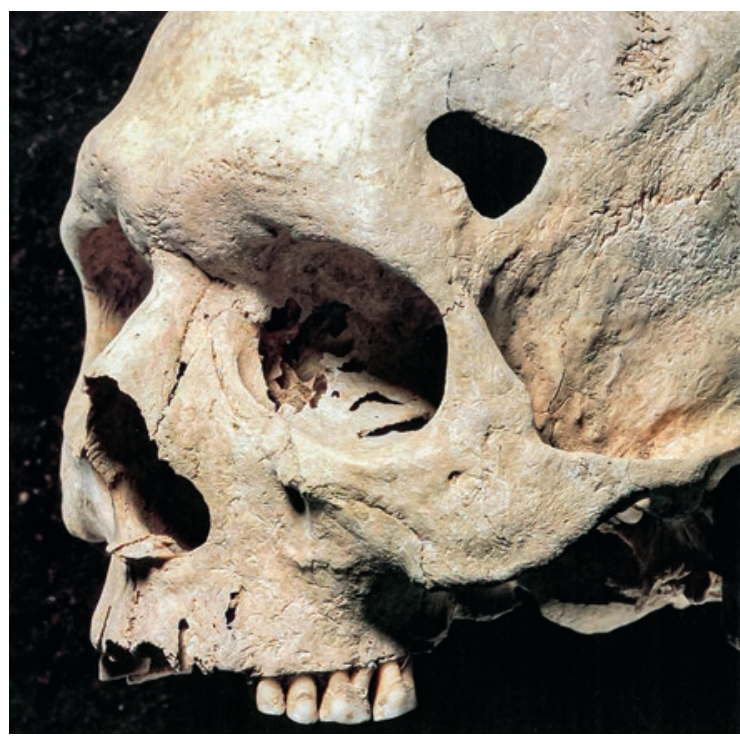

Trepanation, Magleby-Hulbjergbanken (DK), Grabfund. 4. Jt. v. Chr. (๔ Lennart Larsen, Nationalmuseet Kopenhagen).

Literatur

1 Czarnetzki A, Hrsg. Stumme Zeugen ihrer Leiden. Paläopathologische Befunde. Tübingen: Attempto-Verlag; 1996.

2 Interview mit Dorothea Hintermann, Ausstellungskuratorin am Museum für Urgeschichte(n) in Zug. Health Point 2020;(1):28. www. lendenmann.org/arbeitsproben/HealthPoint/HealthPoint_20_1/ HP_20_1_d\%2027.pdf

3 Karg S, Weber E. Heilsam, kleidsam, wundersam - Pflanzen im Alltag der Steinzeitmenschen. Darmstadt: wbg Theiss; 2019.

4 Seifert $M$, et al. Die spätbronzezeitlichen Ufersiedlungen von Zug-Sumpf: die Dorfgeschichte. O.O.: Kantonales Museum für Urgeschichte; 1996.

5 Bleicher N. Steinzeit im Parkhaus. Moderne Archäologie und das unsichtbare Welterbe der Pfahlbauten.o.O.: NZZ Libro; 2018. 